\title{
Health-service Use in Women with Binge Eating Disorders
}

\author{
John Dickerson, MS ${ }^{1,}{ }^{*}$, Lynn DeBar, PhD ${ }^{1}$, Nancy A. Perrin, PhD $^{1}$, Frances Lynch, PhD ${ }^{1}, \mathrm{G}$. \\ Terence Wilson, $\mathrm{PhD}^{2}$, Francine Rosselli, $\mathrm{PhD}^{3}$, Helena C. Kraemer, $\mathrm{PhD}^{4}$, and Ruth $\mathbf{H}$. \\ Striegel-Moore, $\mathrm{PhD}^{3}$ \\ ${ }^{1}$ Center for Health Research, Kaiser Permanente Northwest, Portland, OR, USA \\ ${ }^{2}$ Department of Psychology, Rutgers, The State University of New Jersey, New Brunswick, NJ, \\ USA \\ ${ }^{3}$ Department of Psychology, Wesleyan University, Middletown, CT, USA \\ ${ }^{4}$ Department of Psychiatry and Behavioral Sciences, Stanford University, Stanford, CA, USA
}

\begin{abstract}
Objective-To compare health-care utilization between participants who met DSM-IV criteria for Binge Eating Disorder (BED) and those engaged in Recurrent Binge Eating (RBE) and to evaluate whether objective binge eating (OBE) days, a key measurement for diagnosing BED, predicted health-care costs.
\end{abstract}

Method-We obtained utilization and cost data from electronic medical records to augment patient reported data for 100 adult female members of a large health maintenance organization (HMO) who were enrolled in a randomized clinical trial to treat binge eating.

Results-Total costs did not differ between the BED and RBE groups $(\beta=-0.117, \mathrm{z}=-0.48$, $\mathrm{p}=0.629)$, nor did the number of OBE days predictor total costs $(\beta=-0.017, \mathrm{z}=-1.01, \mathrm{p}=0.313)$.

Conclusions-Findings suggest that the medical impairment, as assessed through health care costs, caused by BED may not be greater than impairment caused by RBE. The current threshold number of two OBE days/week as a criterion for BED may need to be reconsidered

Eating disorders are associated with considerable medical and psychosocial impairment (1). Use of health services represents an important indicator of impairment, as this measurement reflects subjective distress (pain or suffering), dysfunction, or both. The few published studies that have examined health services use among individuals with eating disorders have reported two major findings. First, eating disorders are relatively undertreated. Epidemiological studies consistently show that few participants with eating disorders report having received treatments specifically targeting the eating problem (2-7). Second, despite underutilization of targeted care, health-insurance claims data suggest that total healthservices use and costs are elevated among individuals with eating disorders compared to matched controls $(8-10)$.

This literature is constrained by methodological limitations. The epidemiological studies cited above were not designed to measure specific features or costs of health services use, and the self-report nature of the data collected in these studies limits the reliability of the findings (11). Studies utilizing electronic medical records (EMR) typically cannot verify the diagnostic information; consequently, the validity of the diagnoses is in question. Patients may be seeking treatment for their eating disorder without making explicit to the care

*Address for correspondence: John F. Dickerson, MS, Center for Health Research, Kaiser Permanente Northwest, 3800 N. Interstate Ave., Portland, OR 97227, USA, (Phone: (503) 335-2412, Fax: (503) 335-6311, john.f.dickerson@kpchr.org). 
provider that they suffer from an eating disorder. Even if a care provider correctly recognizes the eating disorder, the clinician may not always assign the proper diagnostic code for reasons such as lack of insurance coverage for eating disorder diagnoses or because the provider may consider the eating disorder secondary to another disorder such as depression.

Previous research found that diagnosis moderated the amount of health services use in eating disorders, with greatest use reported for women with anorexia nervosa (AN) and lowest use for women with Eating Disorders Not Otherwise Specified (EDNOS) (9;12). Data about health services use in individuals with EDNOS is especially needed because this group represented the largest segment of those both meeting diagnostic criteria in epidemiological studies $(13 ; 14)$ and also receiving treatment as reflected in medical record data $(9 ; 15)$.

EDNOS is a "catch-all" diagnostic category including individuals with diverse clinical presentations and most studies examining EDNOS do not provide detailed information about the clinical picture of individuals with this diagnosis (16). The few studies that do report such information suggest that individuals experiencing recurrent binge eating are a sizable subgroup included in EDNOS (17-19). Indeed, binge eating disorder (BED) was introduced in the fourth edition of the Diagnostic and Statistical Manual for Mental Disorders (DSMIV; American Psychiatric Association, 1990) as a specific example of EDNOS. Little is known about health services use in BED, in part because previous studies utilizing EMR data have been based on data sets that did not disaggregate EDNOS into more specific subgroups.

The nosology of eating disorders is being reconsidered as part of the development of the fifth edition of the DSM. The diagnostic threshold of a minimum of two binge eating episodes or days per week for a diagnosis of bulimia nervosa (BN) or binge eating disorder (BED), respectively, has been the focus of considerable debate in light of a growing literature that suggests that this threshold is unduly restrictive; some experts have recommended using a frequency threshold of one bulimic episode per week $(20 ; 21)$. Therefore, the present study recruited participants using a binge eating frequency threshold requiring at least one day per week with an objective bulimic episode over a three-month period, with no periods of binge-free time greater than two weeks. Participants who met this threshold but did not meet diagnostic criteria for BN or BED were given a diagnosis of "recurrent binge eating" (RBE).

The primary aim of the present study was to expand upon the prior literature by describing costs and health care use associated with binge eating disorders (BED and RBE) with the advantage of stronger methodologies involving both objectively measured health services use and rigorous assessment of patients' diagnostic status. As a second aim, we sought to determine whether women with RBE differed from women with BED on health-services use or costs.

\section{METHODS}

\section{Participants and Recruitment}

The study sample included 100 women who subsequently participated in the Binge Eating Self-Help Treatment (BEST) trial examining the efficacy of a brief guided self-help program for binge eating and were diagnosed with RBE or BED at the beginning of the study.

Recruitment and case finding procedures for the BEST trial have been described in detail in previous reports $(22 ; 23)$. In brief, trial participants were recruited via mailings to a random sample of age-eligible HMO members (ages of 18 through 50) or via announcements and brochures placed in HMO clinics. Inclusion required 12 months of continuous HMO 
membership at the time of recruitment. Members with EMR records indicating severe cognitive impairment or current treatment for severe physical illness were excluded from the recruitment pool, as were about 100 members who were registered as objecting to being included in any research studies. One participant was determined to be an outlier in terms of total health-care costs and was excluded from this study. Because of the small number of men in the BEST trial ( $\mathrm{N}=10$; about $8 \%$ of the randomized sample), the present study included only women: 50 with BED, and 50 with RBE.

\section{Instruments and Procedure}

Demographic information \& body mass index (BMI)-Information about participants' gender, race (coded as white yes/no), ethnicity (coded as Hispanic yes/no), age at randomization (coded as age at last birth date), highest educational attainment (coded as high school or less, some college or more), and height and weight (used to calculate BMI) was obtained as part of the initial screening questionnaire used to identify BEST cases.

Eating disorder diagnosis-Eating disorder diagnoses were based on the Eating Disorder Examination (EDE, (24), $12^{\text {th }}$ edition with text edits from the $14^{\text {th }}$ and $15^{\text {th }}$ editions, a semi-structured interview widely considered the "gold standard" for determining presence and severity of an eating disorder. The diagnostic items of the interview were used to determine whether participants met DSM-IV criteria for BN or BED or study criteria for RBE.

Psychiatric comorbidity-The Structured Clinical Interview for DSM-IV Diagnoses Axis I (SCID-I/NP with psychotic screen, (25) was administered to determine current and lifetime diagnoses of major axis I disorders. For the purpose of the present report, SCID information on axis I disorders was coded as "any current comorbid psychiatric diagnosis" (yes/no) and "number of current comorbid psychiatric disorders" (none, one, more than one). In addition, participants were asked to complete the Beck Depression Inventory(26).

Health-services use and costs-Health-services use was examined for the 12 months leading up to the initial assessment visit. We created comprehensive profiles of HMO services from available electronic HMO data. These EMR data accurately represent services paid for by the HMO $(27 ; 28)$. Services included all outpatient visits (including mental-health specialty and other medical care), all inpatient care, medication utilization, laboratory tests, and radiology procedures.

We estimated costs by applying unit costs developed and tested in previous studies $(27 ; 28)$ to the HMO utilization measures, with the final cost variables representing HMO expenditures. Because recruitment spanned two years, costs were adjusted to 2006 dollars.

Costs were categorized into several sub-categories, as well as summed for a total cost measure ("total costs"). Based on a review of diagnoses recorded in the EMR, we created three mutually exclusive outpatient cost categories: "weight and eating disorder," "mental health," and "other" (which included all outpatient care not captured in the two previous cost categories [e.g., ophthalmology, foot clinic]). Outpatient costs did not include medications dispensed from the HMO's pharmacy system. If multiple diagnoses were recorded for a single encounter (e.g., eating disorder and depression), the entire encounter was assigned to the "weight and eating disorder" category.

We collected data on all dispensings of prescription medications within the integrated HMO pharmacy system. The HMO keeps detailed electronic records on every drug dispensing, including generic name, and the US Food and Drug Administration's (FDA) National Drug 
Code (NDC) for human drugs was used to identify psychotropic medications and create the sub-category of mental health related pharmaceutical cost. We also created a total medication cost category (total medication costs"), including mental health-related drugs.

Data analyses-Descriptive health-service use and cost data are reported separately for both the RBE and BED diagnostic categories, as determined from the EDE interview at the initial assessment. Participants with a diagnosis of BED or RBE were compared on basic demographic and clinical characteristics using Chi-square tests for categorical variables and t-tests for continuous variables. We excluded from all analyses one participant deemed an outlier whose total cost was more than eight standard deviations above the mean after confirming that the high costs were not attributable to weight or an eating disorder.

Analysis of cost data requires consideration of the non-normal distributional properties commonly encountered in such data. Costs are typically positively skewed, with a small proportion of people affecting the upper tail of the distribution through costly encounters or high levels of utilization. To compare health services costs among patients with BED versus RBE, we chose to model health-care costs using a generalized linear model regression framework, specifying a gamma response distribution incorporating a log link and controlling for a set of covariates commonly shown to influence healthcare costs (age, BMI, BDI total score, each center coded)(29-31). The gamma model has been shown to be an appropriate method to analyze cost data and to be superior to other methods of modeling healthcare costs(31). All models included robust standard errors to allow for heteroskedasticity in the data. Results were validated using non-parametric bootstrap methods. We also examined health services costs as a function of binge eating frequency, using the gamma model adjusted for age, BMI, and BDI total scores.

\section{RESULTS}

\section{Sample Description}

Table 1 shows the demographic and clinical characteristics of the study sample by EDE diagnostic category. Of the 100 women included in the study, only $4.0 \%(n=4)$ had an eating disorder code (i.e., were diagnosed with an eating disorder by their health care provider) in their electronic records during the 12 months prior to randomization into the study.

Participants in the BED and RBE groups were similar in terms of age, ethnicity, race, and education. BMI was significantly higher among participants with BED than those with RBE $(t(98)=2.12, p=.037, d=.42)$. The two groups did not differ significantly on the dichotomous measure of current Axis I psychiatric comorbidity; however, a greater percentage of individuals with BED had two or more axis 1 disorders compared to those with $\operatorname{RBE}\left(\chi^{2}(2)=6.40, \varphi=.041, f=.25\right)$. Moreover, participants in the BED group had a significantly higher mean BDI score than those in the RBE group $(t(96)=2.33, p=.022, d$ $=.46)$.

\section{Health Services Utilization and Costs in BED and RBE}

As shown in Table 2 almost all participants had some health-services utilization during the 12 months prior to assessment. Over $90 \%$ of the sample received at least one dispensed drug, and more than half of the sample received some type of psychotropic medication. Treatments specifically targeting the eating disorder or their weight problem were provided only in a minority of cases and mental health service use related to non-eating or related concerns also was relatively uncommon. Table 2 also presents descriptive statistics of annual costs by diagnostic category and effects sizes (Cohen's $d$ ) comparing costs between 
the groups. Medians are included along with means to provide additional information about the underlying distribution of costs. In most cases, the median was well below the mean, a reminder of the positively skewed nature of cost data. None of the effect sizes exceeded $\mathrm{d}=$ 0.20 , which is considered the lower threshold for defining a small effect (32). In addition the effect sizes do not suggest a consistent pattern of differences between diagnostic categories as some effect sizes are positive and others negative.

\section{Comparisons of Health-Care Costs in BED and RBE}

Table 3 provides the coefficients and standard errors from the gamma regressions of cost data. The BED and RBE groups did not differ significantly in total costs or in any cost subcategories in analyses adjusted for age, BMI, and self-reported depression (BDI). We did find some expected associations between covariates and healthcare costs: age was positively related to both total medications cost and total costs; higher BMI was significantly associated with higher mental health medication- and total medication costs; and greater depression, as measured by the BDI, was associated with higher total costs. Nonparametric bootstrap estimates found the same pattern of significance as the gamma regressions presented.

\section{Number of Binge Eating Days as a Predictor of Cost}

Additional post-hoc analyses examined whether days with objective bulimic episodes ("binge eating days") a key metric used to diagnose BED, was a linear predictor of costs. As shown in Table 4, the number of binge eating days during the past 28 days did not predict any measure of health-care costs. We also found similar results between covariates (i.e., age, BMI, and BDI scores) and costs as those reported in the previous section. Nonparametric bootstrap estimates found the same pattern of significance as the gamma regressions presented.

\section{DISCUSSION}

This study examined health-services use and costs among female health plan members who had been diagnosed with a binge eating disorder as part of a randomized clinical intervention trial. Our study expanded upon the previous literature in three major ways: first, we determined eating disorder diagnosis using a rigorous diagnostic interview (the EDE); second, we obtained objective measures of health-services use and costs by extracting information from electronic records; and third, we included patients with RBE, thus adding much-needed information to the existing understanding of the clinical significance of eating disturbances that do not meet current diagnostic thresholds.

All but two participants had received health services during the 12 months prior to their baseline assessment for the binge eating treatment trial. About half of these participants had been prescribed medications for mental health conditions, a finding that is not surprising in light of the considerable psychiatric comorbidity observed in our sample. Yet, only a fraction of our sample had been diagnosed with or treated specifically for an eating disorder, suggesting that eating disorders largely go undetected or untreated by health care professionals even in the context of treatment for other mental health problems.

Health-care costs did not differ significantly between BED and RBE in analyses adjusting for differences in age, self-reported depression, and BMI, suggesting no discernible difference in impairment as reflected in the use of health services. We found age, BMI, and self-reported depression to be significant predictors of costs within different cost categories, supporting our rational for including these covariates in all of our models. Although we found a nearly significant association between the number of objective binge eating days 
and weight- and eating disorder-related costs, the number of binge eating days was not associated significantly with any other cost measure. Our results show similar levels of impairment between BED and RBE groups, raising the concern that the current threshold of binge eating frequency used for standard diagnostic criteria may be too conservative. Medical providers often do not recognize any type of eating disorder, and may be particularly unaware of the potentially negative impacts of eating disorders that do not meet criteria for anorexia nervosa or bulimia nervosa. Accordingly, those presenting with subthreshold symptom may be more vulnerable to remaining undetected and untreated.

Our findings support previous research indicating that nonspecific eating disorder diagnoses, such as EDNOS, appear to be associated with similar levels of high health-care utilization as do major ED diagnoses such as BN (10). The lack of difference in health-care costs among individuals with BED versus those with RBE calls into question the clinical utility and significance of current diagnostic frequency thresholds, and supports reconsideration of current diagnostic criteria for EDNOS. In addition, greater attention and awareness of partial and non-specific eating disorders in primary care could aid in providing needed care to persons suffering from these disorders.

Several limitations need to be considered. Our sample proved to be demographically homogeneous. Despite outreach efforts and the availability of Spanish language study materials, few Hispanic health plan members (the largest ethnic minority group in the HMO's geographic area) participated in the BEST study. Because the health plan does not include race or ethnicity data in individual patient records we cannot answer the question of whether Hispanic plan members were disproportionately underrepresented in our sample or whether health services use or costs vary by ethnicity. Also, few men enrolled in the study. Men comprise a minority of individuals who develop eating disorders (33) and are especially unlikely to receive treatment for an eating disorder (9). In BEST, only 10 men were included; therefore, our sample of men was too small for further analyses concerning health services use. Further, an important question that we were not able to address in this sample eligible for inclusion by virtue of their reports of binge eating is whether or not treatment utilization and costs might differ between obese individuals who do not report binge eating and the types of individuals included in this study. Other recent research has also suggested the importance of comparing such clinical findings between these populations (21). Finally, the BEST trial was not powered to compare baseline health services costs between RBE and BED groups, so our inability to detect differences between the two groups may be due to inadequate sample size. However, inspection of effect size estimates supports the interpretation that the group differences in health services use and costs were negligible and that both groups therefore were comparable on our indicators of impairment.

This study benefits from objective information on health services use and diagnoses available from an integrated HMO's electronic medical record system, as well as from the inclusion of both BED and RBE study participants. The latter situation afforded a unique opportunity to test for differences in health-care costs between women with BED and women who engage in recurrent binge eating but do not meet the diagnostic criteria for a BED diagnosis.

We provide empirical evidence that the threshold of binge eating days used in the DSM-IV provisional diagnosis does not differentiate impairment, as measured by health-services use, between patients with BED and patients with RBE. More broadly, our study illustrates that eating disorders tend to go undetected or untreated even among individuals who utilize their health plan services. 


\section{Acknowledgments}

This research was supported by MH066966 (to principal investigator R.S.M.) from the National Institutes of Health (NIH) and by the National Institute of Mental Health (NIMH), and the National Institute of Diabetes and Digestive and Kidney Diseases (NIDDK) (awarded to Kaiser Foundation Research Institute [KFRI]). The contents of this study are solely the responsibility of the authors and do not necessarily represent the official of the NIH, NIMH, NIDDK, or the KFRI. Mr. Dickerson and Drs. DeBar, Perrin, Lynch, Wilson, Rosselli, Kraemer, and StriegelMoore report no competing interests and no commercial or industry support. The BEST trial is registered with ClinicalTrials.gov (Identifier: NCT000158340).

\section{Reference List}

1. Mathers, C.; Vos, T.; Stevenson, C. The Burden of Disease and Injury in Australia. Canberra: Australian Institute of Health and Welfare; 1999. Report No.: AIHW catalogue no. PHE17

2. Alegria M, Woo M, Cao Z, Torres M, Meng XL, Striegel-Moore R. Prevalence and correlates of eating disorders in Latinos in the United States. Int J Eat Disord. 2007 Nov; 40( Suppl):S15-S21. [PubMed: 17584870]

3. Hudson JI, Hiripi E, Pope HG Jr, Kessler RC. The prevalence and correlates of eating disorders in the National Comorbidity Survey Replication. Biol Psychiatry. 2007 Feb 1; 61(3):348-58. [PubMed: 16815322]

4. Mond JM, Hay PJ, Rodgers B, Owen C. Health service utilization for eating disorders: findings from a community-based study. Int J Eat Disord. 2007 Jul; 40(5):399-408. [PubMed: 17497708]

5. Striegel-Moore RH, Dohm FA, Wilfley DE, Pike KM, Bray NL, Kraemer HC, et al. Toward an understanding of health services use in women with binge eating disorder. Obes Res. 2004 May; 12(5):799-806. [PubMed: 15166300]

6. Striegel-Moore RH, Dohm FA, Kraemer HC, Schreiber GB, Crawford PB, Daniels SR. Health services use in women with a history of bulimia nervosa or binge eating disorder. Int J Eat Disord. 2005 Jan; 37(1):11-8. [PubMed: 15690460]

7. Nicdao EG, Hong S, Takeuchi DT. Prevalence and correlates of eating disorders among Asian Americans: results from the National Latino and Asian American Study. Int J Eat Disord. 2007 Nov; 40( Suppl):S22-S26. [PubMed: 17879986]

8. Simon J, Schmidt U, Pilling S. The health service use and cost of eating disorders. Psychol Med. 2005 Nov; 35(11):1543-51. [PubMed: 16219112]

9. Striegel-Moore RH, Leslie D, Petrill SA, Garvin V, Rosenheck RA. One-year use and cost of inpatient and outpatient services among female and male patients with an eating disorder: evidence from a national database of health insurance claims. Int J Eat Disord. 2000 May; 27(4):381-9. [PubMed: 10744844]

10. Striegel-Moore RH, Debar L, Wilson GT, Dickerson J, Rosselli F, Perrin N, et al. Health services use in eating disorders. Psychol Med. 2008 Oct; 38(10):1465-74. [PubMed: 17976250]

11. Marshall R, Grayson D, Jorm A, O’Toole B. Are survey measures of medical care utilisation misleading? A comparison of self-reported medical care consumption with actual medical care utilisation. Aust Health Rev. 2001; 24(3):91-9. [PubMed: 11668933]

12. Striegel-Moore RH, Silberstein LR, Rodin J. Toward an understanding of risk factors for bulimia. Am Psychol. 1986 Mar; 41(3):246-63. [PubMed: 3457546]

13. Walsh BT, Wilson GT, Loeb KL, Devlin MJ, Pike KM, Roose SP, et al. Medication and psychotherapy in the treatment of bulimia nervosa. Am J Psychiatry. 1997 Apr; 154(4):523-31. [PubMed: 9090340]

14. Sysko R, Walsh BT, Fairburn CG. Eating Disorder Examination-Questionnaire as a measure of change in patients with bulimia nervosa. Int J Eat Disord. 2005 Mar; 37(2):100-6. [PubMed: 15732070]

15. Roehrig M, Thompson JK, Brannick M, van den Berg P. Dissonance-based eating disorder prevention program: a preliminary dismantling investigation. Int J Eat Disord. 2006 Jan; 39(1):110. [PubMed: 16254869] 
16. Thomas JJ, Vartanian LR, Brownell KD. The relationship between eating disorder not otherwise specified (EDNOS) and officially recognized eating disorders: meta-analysis and implications for DSM. Psychol Bull. 2009 May; 135(3):407-33. [PubMed: 19379023]

17. Eddy KT, Tanofsky-Kraff M, Thompson-Brenner H, Herzog DB, Brown TA, Ludwig DS. Eating disorder pathology among overweight treatment-seeking youth: Clinical correlates and crosssectional risk modeling. Behav Res Ther. 2007 Apr 6.

18. Fichter MM, Quadflieg N. Long-term stability of eating disorder diagnoses. Int J Eat Disord. 2007 Nov; 40( Suppl):S61-6. S61-S66. [PubMed: 17702021]

19. Rockert W, Kaplan AS, Olmsted MP. Eating disorder not otherwise specified: the view from a tertiary care treatment center. Int J Eat Disord. 2007 Nov; 40( Suppl):S99-S103. S99-S103. [PubMed: 17879988]

20. Wilson GT, Sysko R. Frequency of binge eating episodes in bulimia nervosa and binge eating disorder: Diagnostic considerations. Int J Eat Disord. 2009 Nov; 42(7):603-10. [PubMed: 19610014]

21. Wonderlich SA, Gordon KH, Mitchell JE, Crosby RD, Engel SG. The validity and clinical utility of binge eating disorder. Int J Eat Disord. 2009 Dec; 42(8):687-705. [PubMed: 19621466]

22. DeBar LL, Yarborough BJ, Striegel-Moore RH, Rosselli F, Perrin N, Wilson GT, et al. Recruitment for a guided self-help binge eating trial: potential lessons for implementing programs in everyday practice settings. Contemp Clin Trials. 2009 Jul; 30(4):326-33. [PubMed: 19275947]

23. Striegel-Moore RH, Perrin N, Debar L, Wilson GT, Rosselli F, Kraemer HC. Screening for binge eating disorders using the Patient Health Questionnaire in a community sample. Int J Eat Disord. 2009 May 7.

24. Fairburn CG, Bohn K. Eating disorder NOS (EDNOS): an example of the troublesome "not otherwise specified" (NOS) category in DSM-IV. Behav Res Ther. 2005 Jun; 43(6):691-701. [PubMed: 15890163]

25. First, MB.; Spitzer, RL.; Gibbon, M.; Williams, JB. Structured Clinical Interview for DSM-IV-TR Axis I disorders, research version, non-patient edition, with psychotic screen (SCID-I/NP, w/PSY screen). New York, NY: Biometrics Research, New York Psychiatric Institute; 2002.

26. Beck AT, Steer RA, Garbin MG. Psychometric properties of the Beck Depression Inventory: Twenty-five years of evaluation. Clinical Psychology Review. 1988; 8(1):77-100.

27. Hornbrook MC, Goodman MJ. Chronic disease, functional health status, and demographics: a multi- dimensional approach to risk adjustment. Health Serv Res. 1996 Aug; 31(3):283-307. [PubMed: 8698586]

28. Lynch FL, Hornbrook MC, Clarke GN, Perrin N, Polen M, O’Connor E, et al. Cost-effectiveness of an intervention to prevent depression in at risk teens. Arch Gen Psychiatry. 2005 Nov; 62(11): 1241-8. [PubMed: 16275811]

29. Moran JL, Solomon PJ, Peisach AR, Martin J. New models for old questions: generalized linear models for cost prediction. J Eval Clin Pract. 2007 Jun; 13(3):381-9. [PubMed: 17518803]

30. Montez-Rath M, Christiansen CL, Ettner SL, Loveland S, Rosen AK. Performance of statistical models to predict mental health and substance abuse cost. BMC Med Res Methodol. 2006; 6:53. [PubMed: 17067394]

31. Manning WG, Basu A, Mullahy J. Generalized modeling approaches to risk adjustment of skewed outcomes data. J Health Econ. 2005 May; 24(3):465-88. [PubMed: 15811539]

32. Cohen, J. Statistical power analysis for the behavioral sciences. Hillsdale, New Jersey: Lawrence Erlbaum Associates; 1988.

33. Huang JS, Norman GJ, Zabinski MF, Calfas K, Patrick K. Body image and self-esteem among adolescents undergoing an intervention targeting dietary and physical activity behaviors. J Adolesc Health. 2007 Mar; 40(3):245-51. [PubMed: 17321425] 
Table 1

Demographic characteristics by eating disorder diagnostic category at initial assessment

\begin{tabular}{|l|c|c|c|}
\hline & $\begin{array}{c}\text { BED } \\
\text { N=50 }\end{array}$ & $\begin{array}{c}\text { RBE } \\
\text { N=50 }\end{array}$ & $P^{I}$ \\
\hline Age & $36.4(7.3)$ & $38.6(8.3)$ & 0.158 \\
\hline Body Mass Index & $33.4(6.1)$ & $30.9(5.7)$ & 0.037 \\
\hline Education: some college or more & $82.0 \%$ & $80.0 \%$ & 0.799 \\
\hline White & $100 \%$ & $97.9 \%$ & 0.310 \\
\hline Hispanic & $2.0 \%$ & $6.0 \%$ & 0.307 \\
\hline Beck Depression Inventory & $21.0(8.3)$ & $17.5(6.6)$ & 0.022 \\
\hline Number of comorbid axis 1 disorders & & & \\
\hline 0 & $36.0 \%$ & $42.0 \%$ & 0.041 \\
\hline 1 & $24.0 \%$ & $40.0 \%$ & \\
\hline $2+$ & $40.0 \%$ & $18.0 \%$ & \\
\hline Number of days with an objective bulimic episode in the past 28 days & $15.0(7.1)$ & $9.0(5.9)$ & NA ${ }^{2}$ \\
\hline Number of objective bulimic episodes in the past 28 days & $17.9(11.7)$ & $11.8(11.9)$ & NA \\
\hline
\end{tabular}

$B E D$, Binge Eating Disorder; RBE, Recurrent Binge Eating Disorder (minimum average number of one day per week during the preceding 3 months with an objective bulimic episode with no gaps between episodes of two weeks or more); BMI, body mass index.

Values in parentheses are standard deviations.

$1_{2}$

2-way comparison between BED and RBE using t-test (continuous measure) or Chi-Square (dichotomous measures).

${ }^{2}$ Used to construct RBE and BED diagnostic categories. 


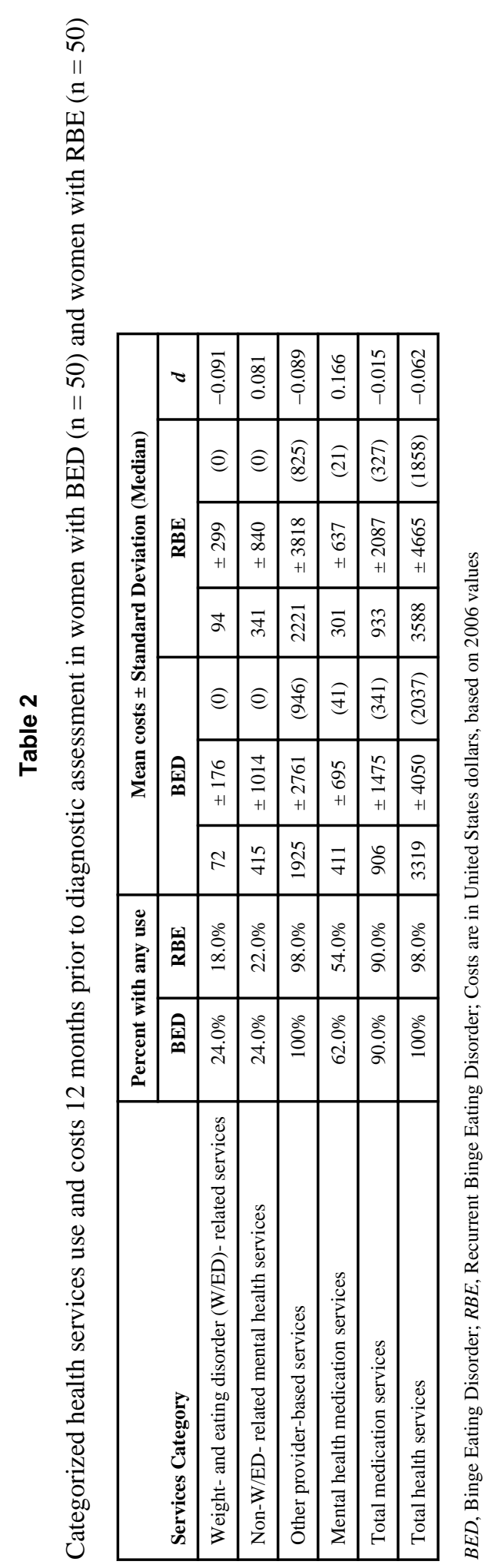

Int J Eat Disord. Author manuscript; available in PMC 2014 February 17. 


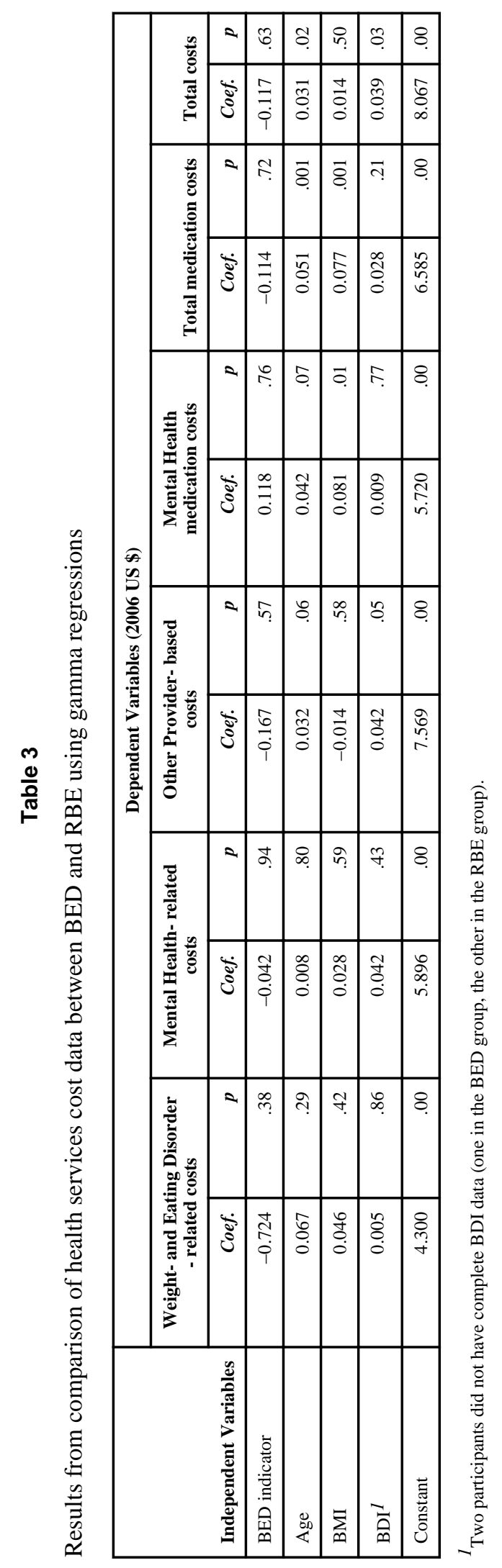

Int J Eat Disord. Author manuscript; available in PMC 2014 February 17. 


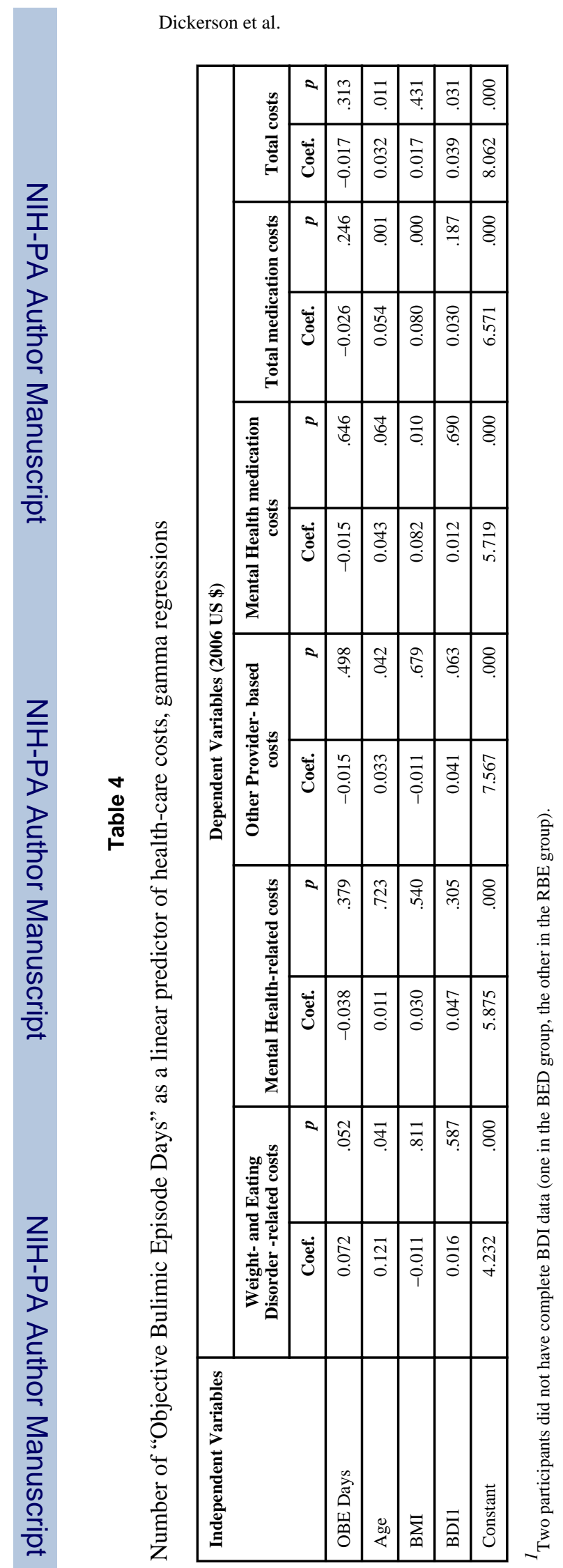

Page 12

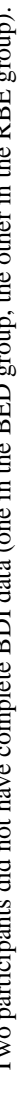

Int J Eat Disord. Author manuscript; available in PMC 2014 February 17. 